\title{
Dysregulation of human NEFM and NEFH mRNA stability by ALS-linked miRNAs
}

Danae Campos-Melo ${ }^{1 \dagger}$, Zachary C. E. Hawley ${ }^{1 \dagger}$ and Michael J. Strong ${ }^{1,2,3,4^{*}}$ (i)

\begin{abstract}
Neurofilaments (NFs) are the most abundant cytoskeletal component of vertebrate myelinated axons. NFs function by determining axonal caliber, promoting axonal growth and forming a 3-dimensional lattice that supports the organization of cytoplasmic organelles. The stoichiometry of NF protein subunits (NFL, NFM and NFH) has to be tightly controlled to avoid the formation of NF neuronal cytoplasmic inclusions ( $\mathrm{NCls}$ ), axonal degeneration and neuronal death, all pathological hallmarks of amyotrophic lateral sclerosis (ALS). The post-transcriptional control of NF transcripts is critical for regulating normal levels of NF proteins. Previously, we showed that miRNAs that are dysregulated in ALS spinal cord regulate the levels of NEFL mRNA. In order to complete the understanding of altered NF expression in ALS, in this study we have investigated the regulation of NEFM and NEFH mRNA levels by miRNAs. We observed that a small group of ALS-linked miRNAs that are expressed in human spinal motor neurons directly regulate NEFM and NEFH transcript levels in a manner that is associated with an increase in NFM and NFH protein levels in ALS spinal cord homogenates. In concert with previous observations demonstrating the suppression of NEFL mRNA steady state levels in ALS, these observations provide support for the hypothesis that the dysregulation of miRNAs in spinal motor neurons in ALS fundamentally alters the stoichiometry of NF expression, leading to the formation of pathological NCls.
\end{abstract}

Keywords: ALS, miRNA, Motor neuron, Neurofilament, NEFM, NEFH

\section{Introduction}

Neurofilaments (NFs) are unique neuron-specific intermediate filaments in vertebrates. They are highly dynamic structures that determine axonal caliber, promote axonal growth and organize the cytoplasm to form a stable 3-dimensional lattice that supports the organization of organelles and cytoplasmic proteins $[1,2]$.

NF subunit proteins (low, medium and high molecular weight neurofilaments; NFL, NFM and NFH, respectively) form homo- and hetero-polymers following a specific stoichiometry and tight spatiotemporal regulation. Conserving NF stoichiometry by controlling the levels of expression of individual NF subunits is critical for the maintenance of healthy neurons.

\footnotetext{
* Correspondence: Michael.Strong@schulich.uwo.ca; mstrong@uwo.ca

${ }^{\dagger}$ Danae Campos-Melo and Zachary C. E. Hawley contributed equally to this work.

${ }^{1}$ Molecular Medicine Group, Robarts Research Institute, Schulich School of Medicine and Dentistry, Western University, London, ON, Canada

${ }^{2}$ Department of Pathology, Schulich School of Medicine and Dentistry,

Western University, London, ON, Canada

Full list of author information is available at the end of the article
}

Alterations of NF mRNA steady stoichiometry and the associated formation of neuronal cytoplasmic inclusions (NCIs) composed of NF proteins are neuropathological markers of degenerating motor neurons in amyotrophic lateral sclerosis (ALS), a progressive neurodegenerative disease [2-4]. Although the exact mechanism by which NF NCIs exert toxicity is unknown, it has been suggested that they alter the internal structure of axons and disrupt axonal transport, in addition to impairing NMDA-mediated calcium influx, compromising the survival of neurons $[4,5]$.

Post-transcriptional control is crucial for preserving NF subunit expression in neuronal homeostasis and also during axonal outgrowth in development and regeneration [6-8]. MiRNAs are evolutionary conserved non-coding RNAs that control the expression of the majority of the mammalian transcriptome and have been increasingly linked to neurodegenerative disorders. We and others have described a profound dysregulation of miRNAs in spinal cord and motor cortex of ALS patients [9-12]. We previously demonstrated that a selective group of these

(C) The Author(s). 2018 Open Access This article is distributed under the terms of the Creative Commons Attribution 4.0 International License (http://creativecommons.org/licenses/by/4.0/), which permits unrestricted use, distribution, and 
miRNAs directly regulate NEFL mRNA stability [9], and postulated that this dysregulation of miRNA expression would contribute to the selective suppression of NEFL mRNA levels observed in ventral lateral spinal cord motor neurons in ALS [13, 14]. Proper control of the levels of the NF triplet is critical because the backbone of the NF is mainly formed by NFL [15] and the stoichiometry of NFL/NFM/NFH (4:2:1) has to be carefully maintained [16]. The miRNAs responsible for regulating human NEFM and NEFH mRNA stability are however unknown. In this study we observed that a limited number of ALS-linked miRNAs that are expressed in spinal motor neurons directly regulate NEFM and NEFH mRNA levels, in a way that might explain the increase in NFM and NFH protein levels that we observed in ALS spinal cords and thus contribute directly to the formation of NF NCIs.

\section{Methods}

\section{Tissue}

Spinal cord samples from sALS patients (median age of death, $60.6+/-3.5$ yrs) and age-matched, neuropathologically healthy control individuals (median age of death, $67.2+/-3.5$ yrs) were used. All ALS cases were both clinically and neuropathologically confirmed using the El Escorial Criteria (World Federation of Neurology Research Group on Neuromuscular Disease, 1994). Written consent for autopsy was obtained from the next of kin at the time of death or from the patient antemortem in accordance with the London Health Sciences Centre consent for autopsy. ALS cases were genotyped and confirmed to have no known mutations in SOD1, TARDBP, FUS or expanded repeats in C9orf72 (Table 1).

\section{3'RACE PCR, cloning and miRNA target prediction}

NEFM and NEFH mRNA 3'UTRs were obtained using 3'RACE PCR. Briefly, TRIzol reagent (Thermo Fisher Scientific) was used for total RNA extraction from human spinal cord tissue. 3'RACE PCR was performed using SMARTer RACE 5'/3' RACE Kit (Takara Bio. Inc., Clontech) and primers hNEFM_3RACE_F1D: 5'CACTTCACACGCCATAGTAAAGGAAGTCACC3' and hNEFH 3RACE_F2: 5'GAGAAGGCCACAGAAG ACAAGGCCGCCAAG3' for NEFM and NEFH 3'U TRs, respectively. 3'UTR isoforms were cloned into pGEMT-Easy vector and sequenced. For luciferase assays, 3'UTRs were subcloned into pmirGLO vector between NheI and SalI sites and linked to the firefly luciferase coding region. Mutations in two nucleotides at the 3'end of each miRNA recognition element (MRE) within the NEFM and NEFH 3'UTRs were made using QuikChange Site-Directed Mutagenesis Kit II (Agilent) according to the manufacturer's instructions. Mutations were carefully designed to ensure no changes were made in the secondary structures of the transcripts using the RNAFold WebServer (http://rna.tbi.univie.ac.at/cgi-bin/ RNAWebSuite/RNAfold.cgi). Both TargetScan (http:// www.targetscan.org/) and miRanda (http://www.microrna.org/microrna/getGeneForm.do) software programs were used to determine miRNAs with predicted MREs in either NEFM or NEFH 3'UTRs.

Table 1 Patient demographics

\begin{tabular}{llllll}
\hline Cases & Gender & Age of symptom onset (years) & Symptom onset & Age of death (years) & Cause of Death \\
\hline C1 & F & - & - & 62 & Heart attack \\
C2 & M & - & - & 74 & Stroke \\
C3 & F & - & - & 68 & NA \\
C4 & M & - & - & 67 & NA \\
C5 & M & - & - & 75 & NA \\
C6 & F & - & - & 74 & Leukemia \\
C7 & M & - & - & 68 & Brain tumor \\
C8 & F & - & - & 53 & Pneumonia \\
A1 & F & 58 & NA & 60 & NA \\
A2 & M & 69 & Upper/lower limbs & 72 & NA \\
A3 & F & 40 & Bulbar & 41 & Systemic failure \\
A4 & M & 55 & NA & 61 & Pneumonia \\
A5 & M & 64 & Upper/lower limbs & 67 & Respiratory failure \\
A6 & M & 69 & Respiratory symptoms & 71 & Respiratory failure \\
A7 & F & 63 & NA & 64 & NA \\
A8 & F & 47 & Bulbar & 49 & Respiratory failure \\
\hline NA N & S & & &
\end{tabular}


miRNA extraction and real-time PCR

Total miRNA extraction using the mirVana miRNA isolation kit (Thermo Fisher Scientific) was performed from human ventral lumbar spinal cord using 5 controls and 8 ALS tissue samples according to the manufacturer's instruction. Yield and purity of the miRNA solution was determined using spectrophotometry while RNA integrity was measured using a bioanalyzer instrument.

MiRNA extracts from the spinal cord of ALS patients or controls were reversed transcribed and then subjected to real-time PCR using the miRCURY LNA $^{\text {tw }}$ Universal RT microRNA PCR (Exiqon) and ExiLENT SYBR Green master mix (Exiqon), according to the manufacturer's instructions. PCRs were performed using the 7900 HT real-time PCR system. Relative expression of miRNAs was normalized to miR-16-5p, a miRNA previously demonstrated to have the same expression in sALS and controls [9]. The analysis of the relative expression of candidate miRNAs between sALS and controls was done using the $\triangle \triangle C T$ method, where fold-change was calculated as $2^{-\Delta \Delta C T}$. All experiments were run in triplicate and significance was determined using Student's $t$-test.

\section{TaqMan real-time PCR}

To examine the expression levels of NEFM and NEFH mRNA, total RNA extraction was performed on 6 ALS patient and 6 control lumbar spinal cord samples using TRIzol reagent (Ambion, Life Technologies). RNA samples were subjected to a cDNA synthesis reaction using the SuperScript IV VILO reverse transcriptase (Invitrogen, Thermo Fisher Scientific) in accordance to the manufactures instructions. Real-time PCR was done on the cDNA templates using the TaqMan Fast Advanced Master Mix and TaqMan Gene Expression Assays (Applied Biosystems, Thermo Fisher Scientific) targeting either NEFM or NEFH. Assays were performed in accordance to the manufactures instructions. TaqMan probes which either targeted NEFM or NEFH were designed with a FAM fluorophore. The expression of $N E F M$ and NEFH were normalized to the expression of a reference gene (HPRT1), which was targeted by a TaqMan probe containing a VIC fluorophore. Changes in NEFM and NEFH mRNA expression between ALS patients and control subjects were determined using the $\triangle \Delta C T$ method, where fold-change was calculated as $2^{-\Delta \Delta C T}$. Experiments were run in triplicate and determined to be significantly different using a Student's $t$-test.

\section{Fluorescent in situ hybridization (FISH)}

To ensure that the miRNAs of interest are expressed in human motor neurons, neuropathologically normal lumbar spinal cord from control subjects was examined for miRNA expression. Tissue sections were formalin-fixed, paraffin embedded (FFPE) and cut into $7 \mu \mathrm{m}$ sections. Samples were UV treated overnight to reduce the lipofuscin-induced auto-fluorescent signal. FISH was performed as described previously (Planell-Sauger et al. 2010). LNA probes were designed with double DIG-labels that targeted the miRNA of interest (Exiqon). DIG-HRP secondary antibody, and Tyramide Signal Amplification (TSA) Systems tagged with a Cy3 fluorophore (PerkinElmer) were used to obtain a fluorescent signal of the miRNA target. Ventral horn of human lumbar spinal cord tissue was examined for positive staining within motor neurons using the Olympus FV1000 confocal microscope.

\section{Cell culture, luciferase assay and relative quantitative RT- PCR}

HEK293T cells were maintained in Dulbecco's Modified Eagle's Medium (DMEM) containing 10\% fetal bovine serum (FBS), at $37^{\circ} \mathrm{C}$ with $5 \% \mathrm{CO}_{2}$. HEK293T cells were plated on 96-well plates with a density of 10,000 cells/ well $24 \mathrm{~h}$ prior to transfection. $100 \mathrm{nM}$ of miRNA mimics (Thermo Fisher Scientific) and $3.47 \mathrm{fmol}$ of pmirGLO containing NEFM or NEFH 3'UTR were co-transfected into the cells using Lipofectamine 2000 reagent (Thermo Fisher Scientific).

Luciferase assays and relative quantitative RT-PCR were performed $24 \mathrm{~h}$ post-transfection as was described previously [17]. Data show positive values as up-regulation and negative values as down-regulation. All experiments were run in triplicate, and significance was determined using a Student's $t$-test or one-way ANOVA followed by Turkey's post hoc test.

\section{Western blot}

Total protein extraction from ventral lumbar spinal cord of 3 controls and 6 ALS patients was performed using NP40 lysis buffer containing proteinase inhibitors. Samples were sonicated, resuspended in loading buffer, denatured at $90{ }^{\circ} \mathrm{C}$ and run on an $8 \%$ SDS-gel. After transfer, the nitrocellulose membrane was probed with either mouse anti-NFM (1:1000; Boehringer Mannheim, 814334), mouse anti-NFH (1:1000; Boehringer Mannheim, 814-342), or rabbit anti-GAPDH (1:5000; Abcam, ab9485) and later with HRP-secondary antibody (goat anti-mouse 1:3000, or goat anti-rabbit 1:5000; BioRad and Invitrogen, respectively). Relative protein expression of NFM and NFH were normalized to GAPDH expression levels. Student's $t$-test was used to determine statistical differences in endogenous protein expression.

\section{Results}

Considering that 3'UTR polymorphisms have been increasingly reported in the literature, we determined if 3'UTR variants of NEFM and NEFH mRNAs are expressed in human spinal cord. A single variant form of 


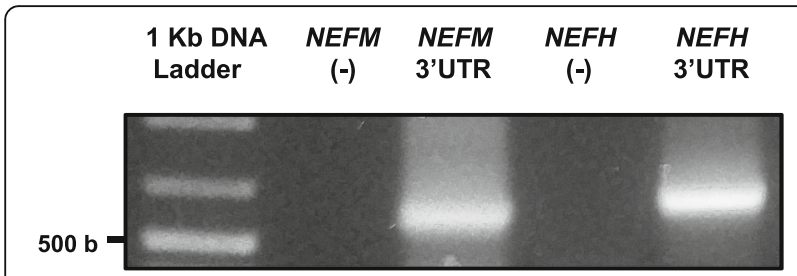

Fig. 1 Single NEFM and NEFH mRNA 3'UTR variants are expressed in human spinal cord. 3'RACE-PCR was performed from spinal cord tissue samples of control individuals using specific primers to amplify NEFM and NEFH 3'UTRs. 3'UTRs were cloned and sequenced. One 3'UTR variant for each NEFM (486 nt) and NEFH (583 nt) transcript was observed in human spinal cord regardless of control or ALS origin of tissue. NEFM specific primer anneals to a region 40 nt upstream the stop codon

NEFM and NEFH 3'UTRs (486 and 583 nt, respectively) was detected in lumbar spinal cord control tissue (Fig. 1). Analysis of ALS patients showed no difference in the 3'UTR variants of NEFM and NEFH expressed in spinal cord compared to control samples (data not shown).

Prediction algorithms showed that NEFM and NEFH 3'UTRs have multiple MREs for different pools of miRNAs. However, for this study we only considered those miRNAs that we previously observed to be differentially expressed in ALS tissue versus controls using the TaqMan assay [9]. We performed real-time
PCR using SYBR green of 40 miRNAs to validate differential expression of 6 miRNAs that have MREs in NEFM or NEFH 3'UTRs (Fig. 2a). Each miRNA, (miR-92a-3p, miR-125b-5p, miR-9-5p, miR-20b-5p and miR-223-3p and miR-519d-3p) showed significant down-regulation of expression in ALS spinal cord versus controls (Fig. 2b).

Next, we examined the neuronal expression of the group of miRNAs that potentially regulate $N E F M$ and $N E F H$ transcripts in human spinal cord motor neurons of control tissue through FISH. MiR-92a-3p is almost exclusively expressed in motor neurons of spinal cord. MiR-125b-5p, miR-9-5p, miR-20b-5p and miR-519d-3p showed higher expression in motor neurons than in other cell types within the spinal cord. MiR-223-3p showed similar expression in motor neurons and surrounding cells. MiR-548c-3p was used as a negative control and miR-124-3p, which is highly expressed in neurons, was used as positive control. In summary, we observed that the 6 ALS-linked miRNAs that are predicted to regulate $N E F M$ and NEFH mRNAs are expressed in motor neurons of human spinal cord (Fig. 3).

Functionality assays of these 6 miRNAs showed that miR-92a-3p and miR-125b-5p down-regulate the levels of a luciferase reporter linked to NEFM 3'UTR (Fig. 4a). MiR-9-5p, miR-20b-5p, miR-92a-3p and miR-223-3p down-regulate the levels of the luciferase reporter

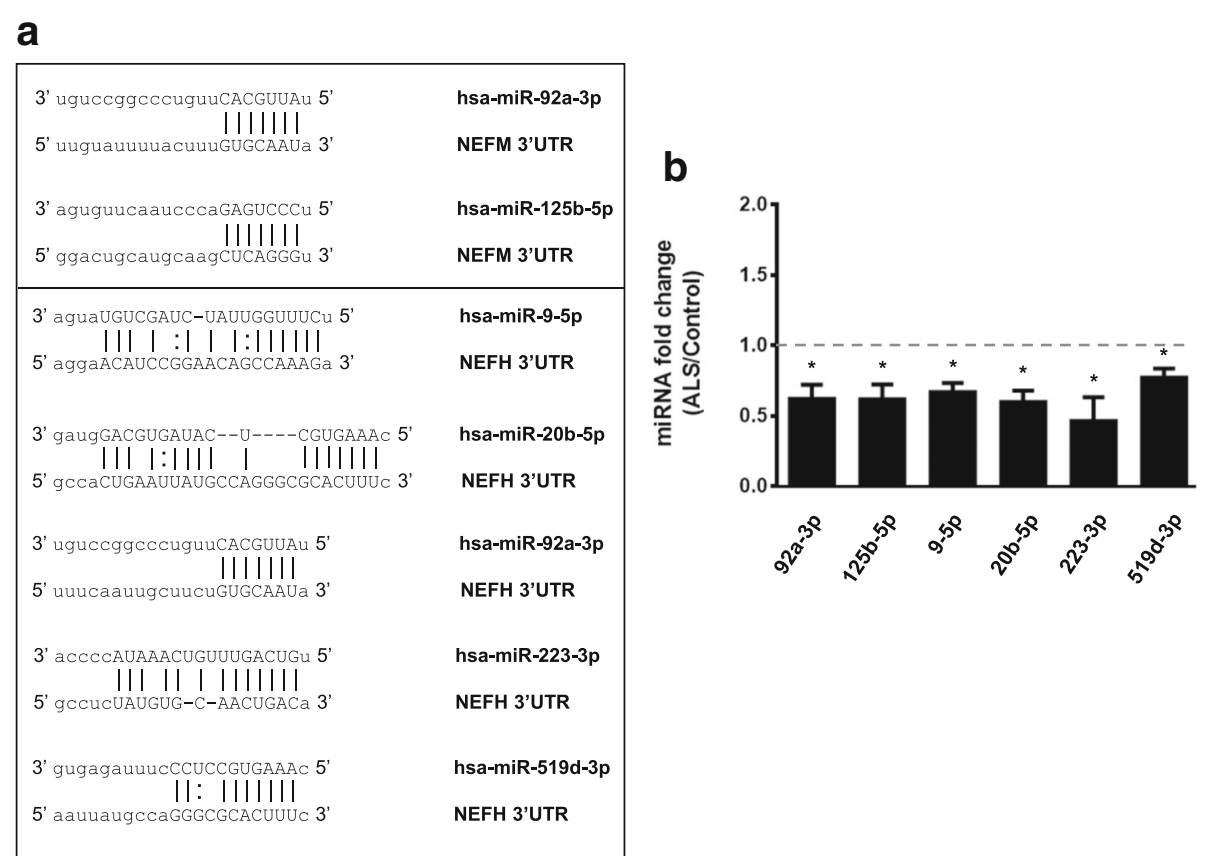

Fig. 2 MiRNAs that have MRES in NEFM or NEFH 3'UTRs are down-regulated in the spinal cord of ALS patients. a MRES within NEFM and NEFH 3'UTRs of ALS-linked miRNAs. b Real-time PCR using SYBR green. Validation of differential expression in ALS versus control spinal cords of 6 miRNAs that have MREs in NEFM or NEFH mRNA 3'UTRs is shown. Experiments were performed in triplicate. Values below 1 indicate downregulation. Results are shown as mean \pm SEM (Student $t$-test: ${ }^{* * *} p<0.0001,{ }^{* * *} p<0.001,{ }^{* *} p<0.01,{ }^{*} p<0.05$. MiR-9-5p, $p=0.0229 ;$ miR20b-5p, $p=0.0230$; miR-92a-3p, $p=0.0234$; miR-125b-5p, $p=0.0412$; miR-223-3p, $p=0.0215$ and miR-519d-3p, $p=0.0232$ ) 

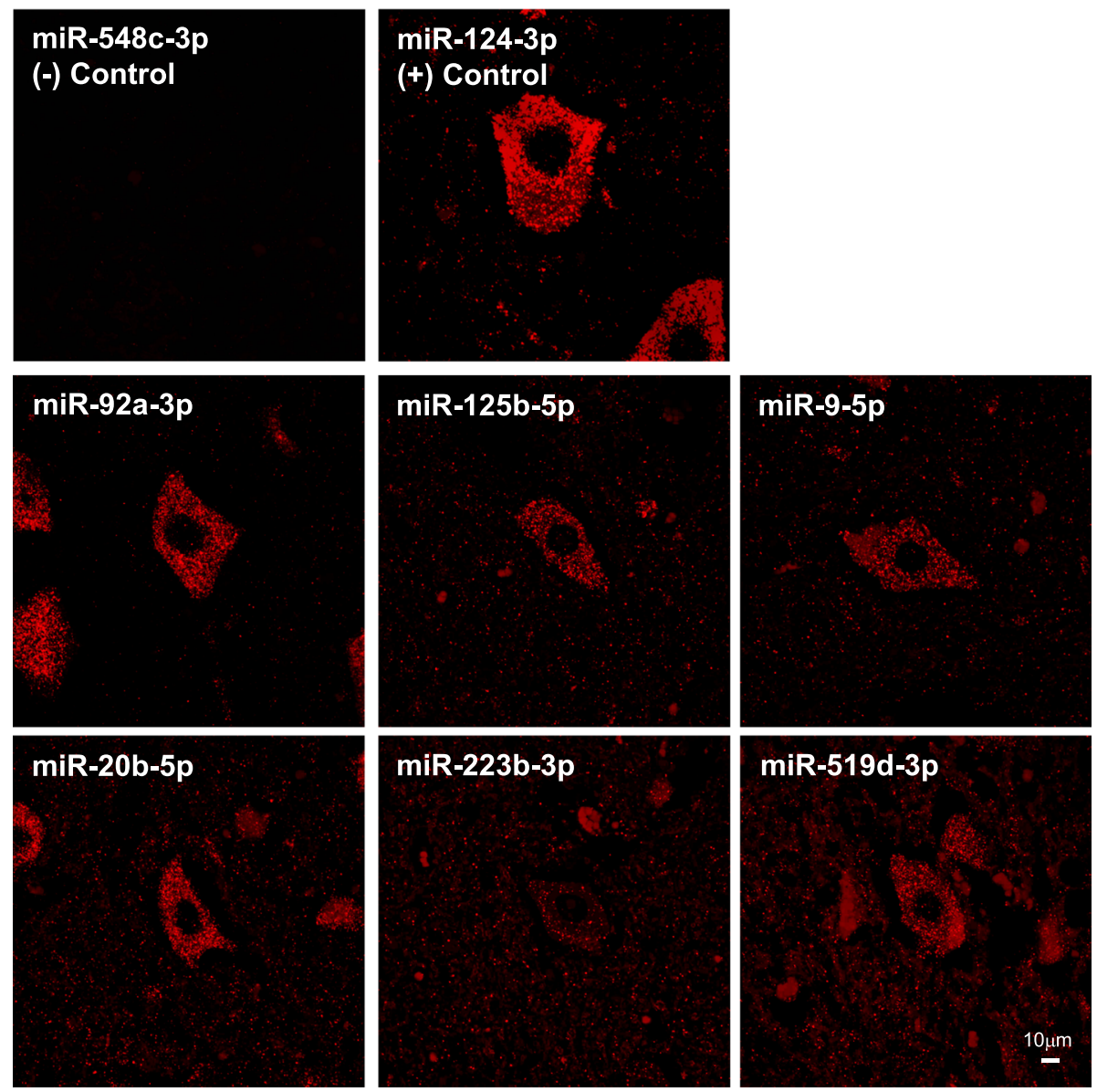

Fig. 3 MiRNAs that have MRES within NEFM or NEFH $3^{\prime} U T R$ T are expressed in motor neurons of human spinal cord control tissue. FISH was performed using FFPE control spinal cord tissue and LNA ${ }^{\mathrm{TM}}$-enhanced detection probes 5'-DIG and 3'-DIG labeled for miRNAs. Amplification was performed using anti-DIG-HRP and TSA Plus Cy3. MiR-548c-3p, which is not expressed in human spinal cord, was used as negative control. MiR124-3p, which is known as highly expressed in neurons, was used as positive control

coupled to NEFH 3'UTR (Fig. 4b). We observed that most of these miRNAs also significantly down-regulate mRNA levels of the luciferase reporter bound to either NEFM or NEFH 3'UTR (Fig. 4c and d), which implies that miRNAs are dysregulating the stability of NEFM and $N E F H$ transcripts. Reporter gene assay using NEFM or NEFH 3'UTR MRE mutants showed a decrease in the down-regulatory effect of each miRNA compared with the wild type, indicating that miR-9-5p, miR-20b-5p, miR-92a-3p, miR-125b-5p and miR-223-3p directly regulate NEFM or NEFH 3'UTRs stability (Fig. 5a and b).

Finally, considering the reduced expression of this group of 5 miRNAs in ALS spinal cords and the down-regulatory function they showed on $N E F M$ and $N E F H$, we should expect an increase of $N E F M$ and $N E F H$ transcript and protein levels in ALS spinal cord tissue compared to controls. Consistent with this, we observed an increase in both $N E F M$ and $N E F H$ transcript and protein levels in ALS ventral lumbar spinal cords (Fig. 6a, b and c).

\section{Discussion}

In this study we have shown that a small group of miRNAs that are dysregulated in the spinal cord of ALS patients directly regulate $N E F M$ and $N E F H$ mRNA stability and that this is associated with an increase in NFM and NFH protein levels in ALS spinal cord homogenates compared to neurological intact control spinal cord homogenates.

The post-transcriptional control of NF transcripts is critical for establishing, consolidating and maintaining normal levels of NF proteins. The stoichiometry of NF subunits has to be tightly controlled to promote axonal outgrowth, control axon caliber and avoid the formation of NF aggregates, axonal degeneration and neuronal death $[4,18]$. The regulation of NF transcripts expression occurs at multiple levels. It has been reported that splicing of the last intron of Xenopus NEFM increases nucleocytoplasmic export of the transcript which allows for robust gene expression [19]. Another level of regulation is at the mRNA transport. One study observed that 


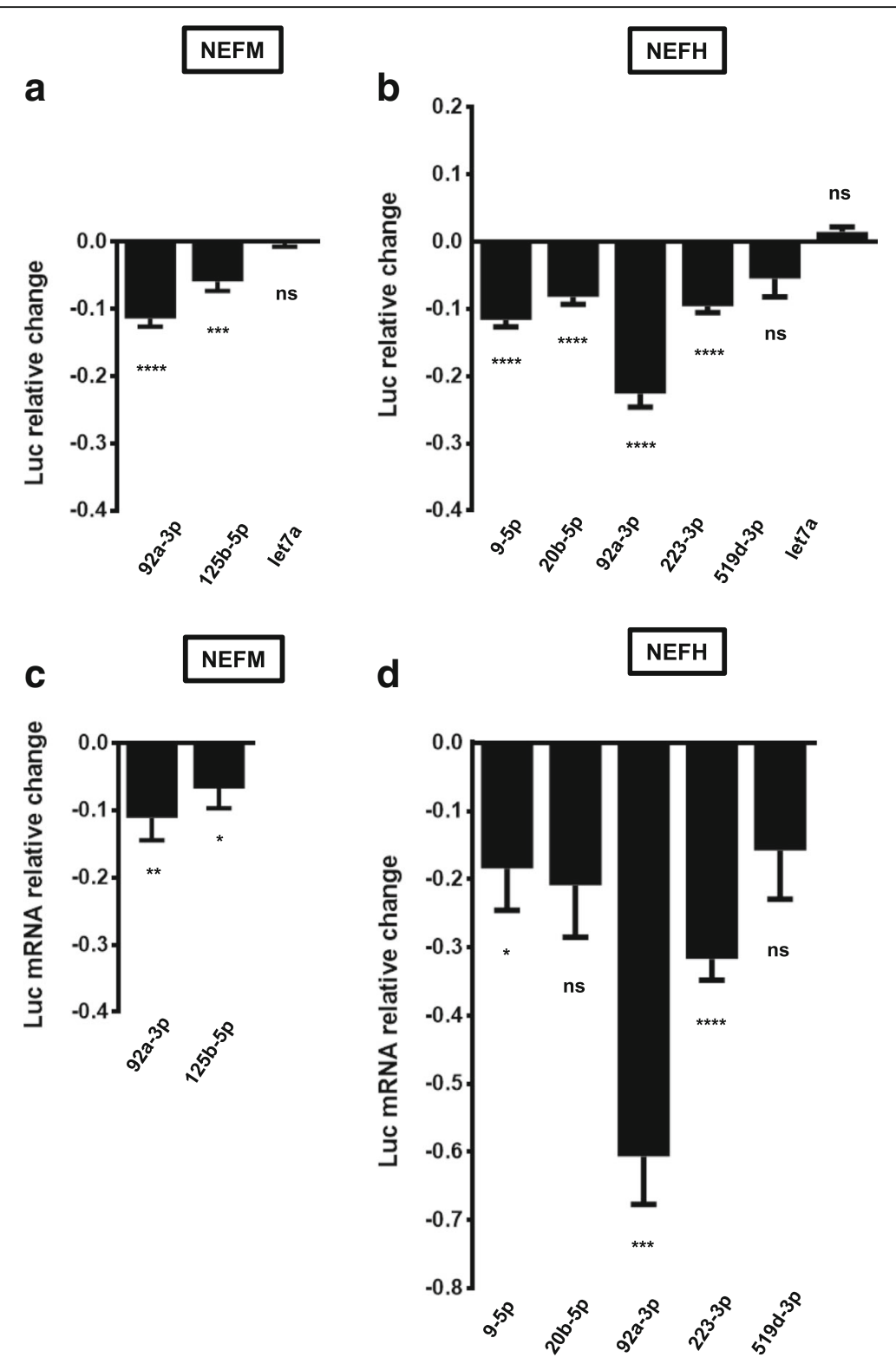

Fig. 4 A group of ALS-linked miRNAs regulate a luciferase reporter linked to NEFM or NEFH 3'UTRs. HEK293T cells were co-transfected with a reporter plasmid containing NEFM or NEFH $3^{\prime} \mathrm{UTRS}$ and miRNA mimics. a, b Reporter gene assays were performed $24 \mathrm{~h}$ after transfection. Data are expressed as relative change and plotted in logarithmic scale. c, $\mathbf{d}$ Relative quantitative RT-PCRs were performed after RNA extraction, $24 \mathrm{~h}$ post-transfection. Data are expressed as relative mRNA level change and plotted in logarithmic scale. All experiments were performed in triplicate. Results are shown as mean \pm SEM (Student $t$-test: **** $p<0.0001,{ }^{* * *} p<0.001,{ }^{* *} p<0.01,{ }^{*} p<0.05$, relative to the pmirGLO vector control). Reporter gene assays: miR-92a-3p/ NEFM, $p<0.0001$; miR-125b-5p/NEFM, $p=0.0005$; miR-let-7a/NEFM, $p=0.6015 ;$ miR-9-5p/NEFH, miR-20b-5p/NEFH, miR-92a-3p/NEFH and miR-223-3p/ NEFH, $p<0.0001 ;$ miR-519d-3p/NEFH, $p=0.0723$; miR-let-7a/NEFH, $p=0.0893$. RT-PCRs: miR-92a-3p/NEFM, $p=0.0052 ;$ miR-125b-5p/NEFM, $p=0.0429 ;$ miR-9-5p/NEFH, $p=0.0431 ; \mathrm{miR}-20 \mathrm{~b}-5 \mathrm{p} / \mathrm{NEFH}, p=0.0518 ; \mathrm{miR}-92 \mathrm{a}-3 \mathrm{p} / \mathrm{NEFH}, p=0.0003 ; \mathrm{miR}-223-3 \mathrm{p} / \mathrm{NEFH}, \mathrm{p}<0.001 ; \mathrm{miR}-519 \mathrm{~d}-3 \mathrm{p} / \mathrm{NEFH}, p=0.0903)$

the mRNAs of each NF subunit are present and translated within intact and regenerating rat sciatic nerve, demonstrating that NF transcripts are transported through axons [20]. At the final stage of mRNA regulation, it has been shown that the RNA-binding protein $\mathrm{HuB}$ increases the translation of NEFM transcript [21].

mRNA stability is the regulatory process of NF transcripts most extensively studied in which multiple trans-acting factors participate. In mice, it has been shown that the RNA-binding protein p190RhoGEF stabilizes and that glycolytic isoenzymes aldolases $\mathrm{A}$ and $\mathrm{C}$ directly destabilizes NEFL mRNA [22, 23]. Our previous studies have shown that the stability of NEFL is regulated by ALS-associated RNA-binding proteins. Mutant copper/zinc superoxide dismutase (mtSOD1) and Rho Guanine Nucleotide Exchange Factor (RGNEF; the 

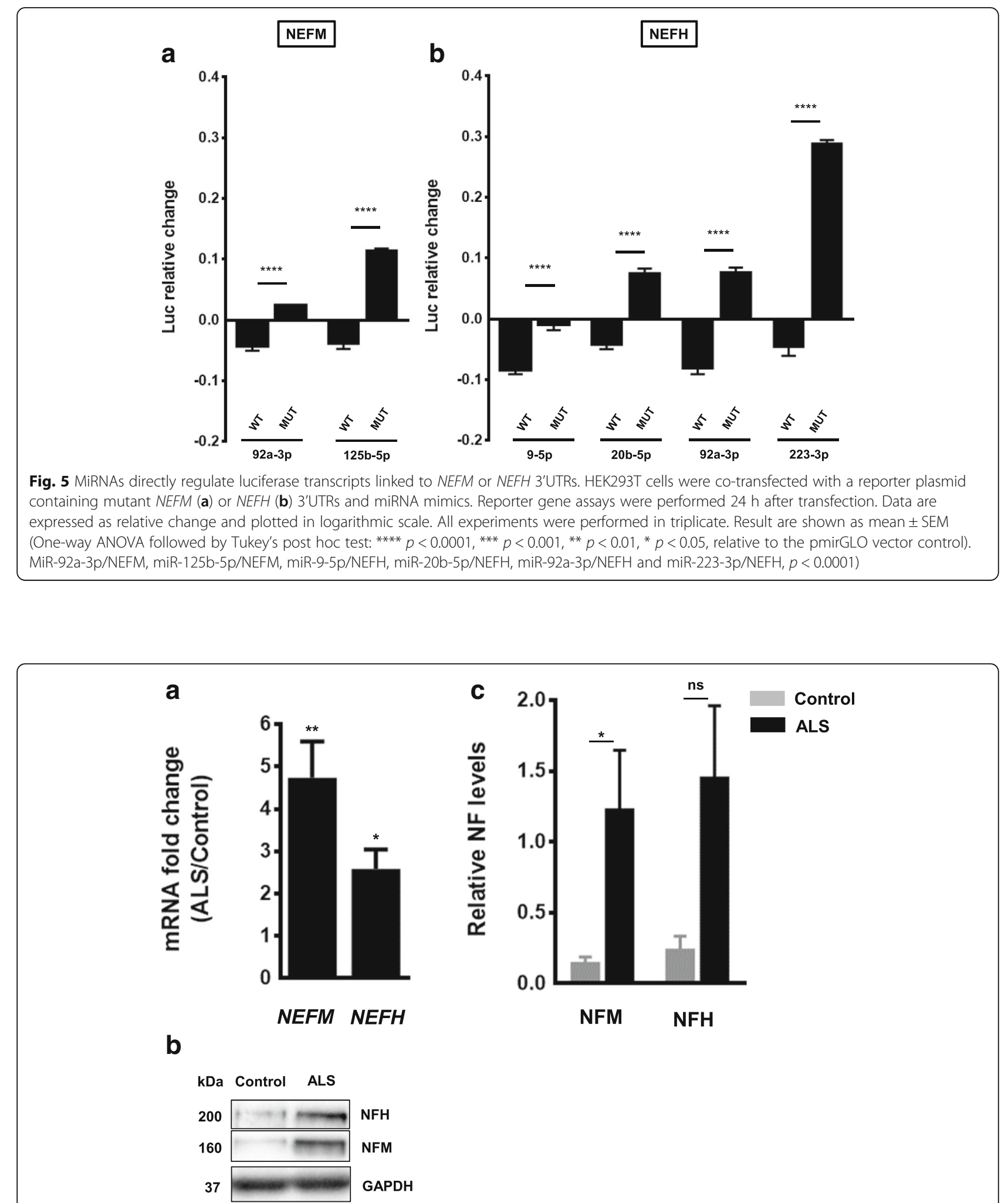

Fig. 6 NEFM and NEFH transcript and protein levels are increased in spinal cord of ALS patients. (a) Real-time PCR using TaqMan and (b) Western blots were performed using ventral lumbar spinal cord samples of controls and ALS patients. (c) Quantification of Western blots in (b). Protein levels were normalized to GAPDH. Data was expressed as the mean \pm SEM (Student $t$-test: ${ }^{* * *} p<0.0001,{ }^{* * *} p<0.001,{ }^{* *} p<0.01,{ }^{*} p<0.05$. Real-time PCR: NEFM, $p=0.0027$ : NEFH, $p=0.0239$. Western blot: NFM, $p=0.0238 ; \mathrm{NFH}, p=0.4762$ ) 
human homologue of p190RhoGEF) mediate the destabilization and TAR DNA binding protein $43 \mathrm{kDa}$ (TDP-43) the stabilization of NEFL mRNA [24-26]. In addition, fused in sarcoma/translocated in liposarcoma (FUS/TLS), another ALS-associated protein, has been shown to bind to murine NEFL, NEFM and NEFH transcripts [27].

The most prominent mechanism of RNA mediated gene silencing involves the interaction of miRNAs with their target mRNAs in which most, but not all, interactions between the miRNA and MREs leads to a degradation of the mRNA. Previously, we and others have observed a massive down-regulation of miRNAs in ALS spinal cord $[11,12,17]$. We also showed that three miRNAs that are dysregulated in ALS, miR-146a*, miR-524-5p and miR-582-3p, regulate levels of NEFL mRNA [9]. In this paper we extended our study to miRNAs responsible for NEFM and NEFH post-transcriptional regulation. We created a list of miRNAs that are down-regulated in ALS spinal cord and that also possess MREs within NEFM and NEFH 3'UTRs. From the published literature, we observed that two miRNAs of this group (miR-9 and miR-125b-5p) were confirmed to be reduced in ALS spinal cord $[11,12]$. We established that a small group of ALS-linked miRNAs (miR-9-5p, miR-20b-5p, miR-92a-3p, miR-125b-5p and miR-223-3p) directly down-regulate human NEFM and $N E F H$ mRNA levels, an effect that is translated into a reduction of NFM and NFH protein levels within spinal cord homogenates. From this group of miRNAs that regulate NEFM and NEFH mRNA levels, only miR-9 has been reported to have a role in neuronal function. More specifically, by regulating several targets including $O C 1$, FoxP1, $M A P 1 B$, and MCPIP1, miR-9 is critical for motor neuron development, function and survival [28].

As these group of miRNAs that regulate NFM and NFH are reduced in spinal cord of ALS tissue, we predicted that the net effect would be an increase of NFM and NFH protein levels in ALS-spinal cords. Several groups have shown that NFL, NFM and/or p-NFH levels are increased in biological fluids of ALS patients [29-33], but there are no reports of NFs protein levels in spinal cord tissue. In this study we showed that both NFM and NFH levels are increased in ventral lumbar spinal cord of ALS patients compared to controls. This observation is in agreement with the increased in NEFM and NEFH transcripts in ALS ventral lumbar spinal cord homogenates that we observed here using real-time PCR and reported previously using RNase protection assay [34].

A selective reduction of NEFL steady state mRNA levels in spinal motor neurons of ALS patients has been well documented [13, 14, 35], a finding that we have proposed is due to alterations in the expression of in NEFL-linked miRNAs [9]. In concert with the observations of this study, we hypothesize that in ALS spinal cords the sustained dysregulation in time of the expression of groups of miRNAs that control NF levels fundamentally alters the expression of all three $N F$ transcripts in a manner that induces an alteration in the stoichiometry of the individual NF proteins, favoring the formation of pathological NCIs.

While this hypothesis supports the critical role of the alteration of miRNA expression in ALS, miRNAs alone are not the sole mediators of RNA stability. Indeed, understanding the fundamental relationship between alterations in RNA-binding proteins and how this interacts with alterations in miRNAs expression will be critical to understanding the process of perturbed RNA-mediated gene silencing which appears to lie at the core of a majority of ALS cases.

\section{Abbreviations}

ALS: Amyotrophic lateral sclerosis; C9orf72: Gene encoding chromosome 9 open reading frame 72; FFPE: Formalin-fixed, paraffin embedded;

FISH: Fluorescent in situ hybridization; FoxP1: Forkhead Box P1; FUS: Gene encoding RNA processing protein fused in sarcoma/translocated in liposarcoma; FUS/TLS: RNA processing protein fused in sarcoma/translocated in liposarcoma; GAPDH: Glyceraldehyde 3-phosphate dehydrogenase; MAP1B: Microtubule associated protein 1B; MCPIP1: Zinc finger CCCH-Type containing 12A; MRE: MiRNA recognition element; mtSOD1: Mutant SOD1; NCls: Neuronal cytoplasmic inclusions; NEFH: High molecular weight neurofilament mRNA; NEFL: Low molecular weight neurofilament mRNA; NEFM: Medium molecular weight neurofilament mRNA; NF: Neurofilament; NFH: High molecular weight neurofilament; NFL: Low molecular weight neurofilament; NFM: Medium molecular weight neurofilament; NMDA: Nmethyl-D-aspartate; OC1: Transcription factor onecut1; RACE: Rapid amplification of CDNA ends; RGNEF: Rho guanine nucleotide exchange factor; SOD1: Gene encoding Cu/Zn superoxide dismutase 1; TARDBP: Gene encoding TAR DNA binding protein $43 \mathrm{kDa}$; TDP-43: TAR DNA binding protein 43 kDa; TSA: Tyramide Signal Amplification; UTR: Untranslated region

\section{Acknowledgements}

We would like to acknowledge Dr. Cristian Droppelmann for critical reading of the manuscript.

\section{Funding}

ERA-Net for Research Programmes on Rare Diseases (E-Rare) and ALS Society of Canada.

\section{Availability of data and materials}

Datasets used in this study are available from the corresponding author upon request.

\section{Authors' contributions}

DCM conceived the experiments. DCM and $\mathrm{ZH}$ performed the experiments and analyzed the data included in the manuscript. DCM, ZH and MJS wrote the manuscript. All authors read and approved the final manuscript.

\section{Ethics approval and consent to participate}

Research on human tissue was approved by "The University of Western Ontario Research Ethics Board for Health Sciences Research Involving Human Subjects (HSREB)".

\section{Consent for publication}

Not applicable.

\section{Competing interests}

The authors declare that this research in done in the absence of any financial and commercial relationships that could be interpreted as a potential competing interest. 


\section{Publisher's Note}

Springer Nature remains neutral with regard to jurisdictional claims in published maps and institutional affiliations.

\begin{abstract}
Author details
${ }^{1}$ Molecular Medicine Group, Robarts Research Institute, Schulich School of Medicine and Dentistry, Western University, London, ON, Canada. ${ }^{2}$ Department of Pathology, Schulich School of Medicine and Dentistry, Western University, London, ON, Canada. ${ }^{3}$ Department of Clinical Neurological Sciences, Schulich School of Medicine and Dentistry, Western University, London, ON, Canada. ${ }^{4}$ University Hospital, LHSC, Rm C7-120, 339, Windermere Road, London, ON N6A 5A5, Canada.
\end{abstract}

Received: 24 April 2018 Accepted: 3 July 2018

Published online: 20 July 2018

\section{References}

1. Walker KL, Yoo HK, Undamatla J, Szaro BG. Loss of neurofilaments alters axonal growth dynamics. J Neurosci. 2001;21:9655-66.

2. Szaro BG, Strong MJ. Post-transcriptional control of neurofilaments: new roles in development, regeneration and neurodegenerative disease. Trends Neurosci. 2010;33:27-37.

3. Xiao S, McLean J, Robertson J. Neuronal intermediate filaments and ALS: a new look at an old question. Biochim Biophys Acta. 2006;1762:1001-12.

4. Thyagarajan A, Strong MJ, Szaro BG. Post-transcriptional control of neurofilaments in development and disease. Exp Cell Res. 2007;313:2088-97.

5. Sanelli TR, Sopper MM, Strong MJ. Sequestration of nNOS in neurofilamentous aggregate bearing neurons in vitro leads to enhanced NMDA-mediated calcium influx. Brain Res. 2004:1004:8-17.

6. Schwartz ML, Shneidman PS, Bruce J, Schlaepfer WW. Stabilization of neurofilament transcripts during postnatal development. Brain Res Mol Brain Res. 1994;27:215-20.

7. Ananthakrishnan L, Gervasi C, Szaro BG: Dynamic regulation of middle neurofilament RNA pools during optic nerve regeneration. Neuroscience 2008, 153:144-153.

8. Ananthakrishnan L, Szaro BG. Transcriptional and translational dynamics of light neurofilament subunit RNAs during Xenopus laevis optic nerve regeneration. Brain Res. 2009;1250:27-40.

9. Campos-Melo D, Droppelmann CA, He Z, Volkening K, Strong MJ. Altered microRNA expression profile in amyotrophic lateral sclerosis: a role in the regulation of NFL mRNA levels. Mol Brain. 2013;6:26.

10. Wakabayashi K, Mori F, Kakita A, Takahashi H, Utsumi J, Sasaki H. Analysis of microRNA from archived formalin-fixed paraffin-embedded specimens of amyotrophic lateral sclerosis. Acta Neuropathol Commun. 2014:2:173.

11. Emde A, Eitan C, Liou LL, Libby RT, Rivkin N, Magen I, Reichenstein I, Oppenheim H, Eilam R, Silvestroni A, et al. Dysregulated miRNA biogenesis downstream of cellular stress and ALS-causing mutations: a new mechanism for ALS. EMBO J. 2015:34:2633-51.

12. Figueroa-Romero C, Hur J, Lunn JS, Paez-Colasante X, Bender DE, Yung R, Sakowski SA, Feldman EL. Expression of microRNAs in human post-mortem amyotrophic lateral sclerosis spinal cords provides insight into disease mechanisms. Mol Cell Neurosci. 2016;71:34-45.

13. Wong NK, He BP, Strong MJ. Characterization of neuronal intermediate filament protein expression in cervical spinal motor neurons in sporadic amyotrophic lateral sclerosis (ALS). J Neuropathol Exp Neurol. 2000;59:972-82

14. Bergeron C, Beric-Maskarel K, Muntasser S, Weyer L, Somerville MJ, Percy ME. Neurofilament light and polyadenylated mRNA levels are decreased in amyotrophic lateral sclerosis motor neurons. J Neuropathol Exp Neurol. 1994:53:221-30

15. Leermakers FA, Zhulina EB. How the projection domains of NF-L and alphainternexin determine the conformations of NF-M and NF-H in neurofilaments. Eur Biophys J. 2010;39:1323-34.

16. Scott D, Smith KE, O'Brien BJ, Angelides KJ. Characterization of mammalian neurofilament triplet proteins. Subunit stoichiometry and morphology of native and reconstituted filaments. J Biol Chem. 1985;260:10736-47.

17. Campos-Melo D, Droppelmann CA, Volkening K, Strong MJ. Comprehensive luciferase-based reporter gene assay reveals previously masked upregulatory effects of miRNAs. Int J Mol Sci. 2014;15:15592-602.

18. Julien JP. Neurofilament functions in health and disease. Curr Opin Neurobiol. 1999;9:554-60
19. Wang C, Szaro BG. Using Xenopus embryos to study transcriptional and posttranscriptional gene regulatory mechanisms of intermediate filaments. Methods Enzymol. 2016;568:635-60.

20. Sotelo-Silveira JR, Calliari A, Kun A, Benech JC, Sanguinetti C, Chalar C, Sotelo JR. Neurofilament mRNAs are present and translated in the normal and severed sciatic nerve. J Neurosci Res. 2000;62:65-74.

21. Antic D, Lu N, Keene JD. ELAV tumor antigen, Hel-N1, increases translation of neurofilament M mRNA and induces formation of neurites in human teratocarcinoma cells. Genes Dev. 1999:13:449-61.

22. Canete-Soler R, Wu J, Zhai J, Shamim M, Schlaepfer WW. p190RhoGEF binds to a destabilizing element in the $3^{\prime}$ untranslated region of light neurofilament subunit mRNA and alters the stability of the transcript. J Biol Chem. 2001:276:32046-50

23. Canete-Soler R, Reddy KS, Tolan DR, Zhai J. Aldolases a and C are ribonucleolytic components of a neuronal complex that regulates the stability of the light-neurofilament mRNA. J Neurosci. 2005;25:4353-64.

24. Ge WW, Wen W, Strong W, Leystra-Lantz C, Strong MJ. Mutant copper-zinc superoxide dismutase binds to and destabilizes human low molecular weight neurofilament mRNA. J Biol Chem. 2005;280:118-24.

25. Strong MJ, Volkening K, Hammond R, Yang W, Strong W, Leystra-Lantz C, Shoesmith C. TDP43 is a human low molecular weight neurofilament (hNFL) mRNA-binding protein. Mol Cell Neurosci. 2007:35:320-7.

26. Droppelmann CA, Keller BA, Campos-Melo D, Volkening K, Strong MJ. Rho guanine nucleotide exchange factor is an NFL mRNA destabilizing factor that forms cytoplasmic inclusions in amyotrophic lateral sclerosis. Neurobiol Aging. 2013;34:248-62.

27. Lagier-Tourenne C, Polymenidou M, Hutt KR, Vu AQ, Baughn M, Huelga SC, Clutario KM, Ling SC, Liang TY, Mazur C, et al. Divergent roles of ALS-linked proteins FUS/TLS and TDP-43 intersect in processing long pre-mRNAs. Nat Neurosci. 2012;15:1488-97.

28. Hawley ZCE, Campos-Melo D, Droppelmann CA, Strong MJ. MotomiRs: miRNAs in motor neuron function and disease. Front Mol Neurosci. 2017;10:127.

29. Rosengren LE, Karlsson JE, Karlsson JO, Persson LI, Wikkelso C. Patients with amyotrophic lateral sclerosis and other neurodegenerative diseases have increased levels of neurofilament protein in CSF. J Neurochem. 1996;67: 2013-8.

30. Ganesalingam J, An J, Bowser R, Andersen PM, Shaw CE. pNfH is a promising biomarker for ALS. Amyotroph Lateral Scler Frontotemporal Degener. 2013;14:146-9.

31. Xu Z, Henderson RD, David M, McCombe PA. Neurofilaments as biomarkers for amyotrophic lateral sclerosis: a systematic review and meta-analysis. PLoS One. 2016;11:e0164625.

32. Feneberg $E$, Oeckl $P$, Steinacker $P$, Verde F, Barro C, Van Damme P, Gray E, Grosskreutz J, Jardel C, Kuhle J, et al. Multicenter evaluation of neurofilaments in early symptom onset amyotrophic lateral sclerosis. Neurology. 2018;90:e22-30

33. Haggmark A, Mikus M, Mohsenchian A, Hong MG, Forsstrom B, Gajewska B, Baranczyk-Kuzma A, Uhlen M, Schwenk JM, Kuzma-Kozakiewicz M, Nilsson P. Plasma profiling reveals three proteins associated to amyotrophic lateral sclerosis. Ann Clin Transl Neurol. 2014;1:544-53.

34. Strong MJ, Leystra-Lantz C, Ge WW. Intermediate filament steady-state mRNA levels in amyotrophic lateral sclerosis. Biochem Biophys Res Commun. 2004;316:317-22.

35. Menzies FM, Grierson AJ, Cookson MR, Heath PR, Tomkins J, Figlewicz DA Ince PG, Shaw PJ. Selective loss of neurofilament expression in $\mathrm{Cu} / \mathrm{Zn}$ superoxide dismutase (SOD1) linked amyotrophic lateral sclerosis. J Neurochem. 2002;82:1118-28. 